\title{
Caracterização clínica e laboratorial de cães naturalmente infectados com Trypanosoma cruzi no semiárido nordestino ${ }^{1}$
}

\author{
Vanessa L. Santana², Almir P. Souza ${ }^{2}$, Dayanne A.S.D. Lima², Ana L. Araújo ${ }^{2}$, Soraia \\ V. Justiniano ${ }^{2}$, Raiara P. Dantas ${ }^{2}$, Paulo M.M. Guedes ${ }^{3}$ e Márcia A. Melo ${ }^{2}$
}

\begin{abstract}
Santana V.L., Souza A.P., Lima D.A.S.D., Araújo A.L., Justiniano S.V., Dantas R.P., Guedes P.M.M. \& Melo M.A. 2012. [Clinical and laboratorial characterization of naturally infected Trypanosoma cruzi dogs in the northeastern semi-arid.] Caracterização clínica e laboratorial de cães naturalmente infectados com Trypanosoma cruzi no semiárido nordestino. Pesquisa Veterinária Brasileira 32(6):536-541. Unidade Acadêmica de Medicina Veterinária, Universidade Federal de Campina Grande, Av. Universitária s/n, Bairro Santa Cecília, Patos, PB 58708-110, Brazil. E-mail: almir@cstrufcg.edu.br

This study aimed to evidence the clinical and laboratorial signs of this disease to help characterize this illness in a natural way in the semiarid in the northeastern region. We evaluated 10 positive for Trypanosoma cruzi dogs, that were identified by serological analysis of immunofluorescence assay (RIFI) and enzyme linked immunosorbent assay (ELISA); molecular analysis by polymerase chain reaction (PCR), direct microscopy and blood culture. The chagasic dogs underwent physical examination, electrocardiographic, radiographic, blood pressure, hematology (erythrocyte and leukocyte count) and biochemical exams (urea, creatinine, ALT, AST, PT, albumin, globulin, CK, CK-MB, and cTnl). The physical examination and the blood pressure were presented within the normal range, while in the electrocardiography the FC was observed as normal with a sinus rhythm, with the exception of one dog that presented a sinus tachycardia (168 bat $/ \mathrm{min}$ ). In the ECG of eight dogs there was increase of duration of $P(47 \pm 6.5 \mathrm{~ms})$ suggestive to atrial enlargement, not confirmed in the radiography. A supraunlevelling was observed in the ST segment in one dog. In the hematological results, thrombocytopenia $\left(187.4 \times 10^{3} \pm 137.2 \times 10^{3}\right)$ and anemia $\left(5.0 \times 10^{6} \pm 1.39 \times 10^{6} / \mathrm{ul}\right)$ were noted. The mean hemoglobin $(11 \pm 2.7 \mathrm{~g} / \mathrm{dL})$, hematocrit $(34 \pm 10.5 \%)$ were below normal limits. The white series were within normal variation, with the exception of eosinophilia observed in three dogs. Individually, there were two dogs which registered leukocytosis, lymphocytosis and neutrophilia. In the biochemical evaluation there was hyperproteinemia PT $=7.2 \pm 0.9 \mathrm{~g} / \mathrm{dL}$, hypoalbuminemia $(2.2 \pm 0.4 \mathrm{~g} / \mathrm{dL})$, hyperglobulinemia $(5.1 \pm 1.0 \mathrm{~g} / \mathrm{dL})$, increased of CK $(196+171 \mathrm{U} / \mathrm{L})$ and there was no alteration on ALT and AST enzymes. The CK-MB isoenzymes and CTnI did not change, except in three dogs. We conclude that dogs naturally infected in the northeastern semiarid present characteristics related to indeterminate chronic form (asymptomatic dogs) and that the identification of the naturally infected dogs with no pathognomonic characteristics of the Chagas disease underscores the importance of this illness in the diagnostic process with the other profiles that show nonspecific or not associated to cardiovascular disease.
\end{abstract}

INDEX TERMS: Heart disease, parasite, Trypanosoma cruzi, Chagas disease, diseases of dog, parasitology.

\footnotetext{
${ }^{1}$ Recebido em 9 de setembro de 2011.

Aceito para publicação em 31 de dezembro de 2011.

${ }^{2}$ Programa de Pós-Graduação em Medicina Veterinária, Unidade Acadêmica de Medicina Veterinária (UAMV), Universidade Federal de Campina Grande (UFCG), Campus de Patos, Avenida Universitária s/n, Bairro
}

Santa Cecília, Patos, PB 58708-110, Brasil. *Autor para correspondência: almir@cstr.ufcg.edu.br.

${ }^{3}$ Departamento de Microbiologia e Parasitologia, Centro de Biociências, Campus Universitário, Universidade Federal do Rio Grande do Norte, Av. Salgado Filho s/n, Natal, RN 59078-900, Brasil. E-mail: pauloguedes@cb.ufrn.br 
RESUMO.- Objetivou-se, com este estudo, evidenciar os sinais clínicos e laboratoriais desta enfermidade para auxiliar na caracterização da doença de forma natural na área semi-árida da região nordeste. Foram avaliados 10 cães positivos para Trypanosoma cruzi, identificados mediante análises sorológicas de reação de imunofluorescência indireta (RIFI) e enzyme linked immunosorbent assay (ELISA); análise molecular pela Reação em Cadeia Polimerase (PCR), microscopia direta e hemocultura. Os cães chagásicos foram submetidos à avaliação física, verificação da pressão arterial, exames eletrocardiográficos, radiográficos, hematológicos (eritrograma e leucograma) e bioquímicos (ureia, creatinina, ALT, AST, PT, albumina, globulina, CK, CK-MB e cTnI). 0 exame físico e os valores das pressões arteriais dos cães apresentaram dentro dos parâmetros de normalidade, enquanto que na eletrocardiografia observou-se FC normal com ritmo sinusal, com exceção de um cão, que apresentou taquicardia sinusal (168 bat/min). No ECG de oito cães houve aumento da duração de $\mathrm{P}(47 \pm 6,5 \mathrm{~ms})$ sugestivo de aumento atrial, não confirmado radiograficamente. Foi observado supradesnivelamento do segmento ST em um cão. Nos resultados hematológicos constatou-se trombocitopenia $\left(187,4 \times 10^{3} \pm 137,2 \times 10^{3}\right)$ e anemia $\left(5,0 \times 10^{6} \pm 1,39 \times 10^{6} /\right.$ $\mathrm{uL})$. Os valores médios da hemoglobina $(11 \pm 2,7 \mathrm{~g} / \mathrm{dL})$ e do hematócrito $(34 \pm 10,5 \%)$ estavam abaixo dos limites de normalidade. A série branca apresentou-se dentro dos limites de normalidade, com exceção da eosinofilia observada em três cães. Individualmente, registrou-se em dois cães, leucocitose, linfocitose e neutrofilia. Na avaliação bioquímica, registrou-se hiperproteinemia $(7,2 \pm 0,9 \mathrm{~g} / \mathrm{dL})$, hipoalbuminemia $(2,2 \pm 0,4 \mathrm{~g} / \mathrm{dL})$, hiperglobulinemia $(5,1 \pm 1,0 \mathrm{~g} / \mathrm{dL})$ e aumento da CK $(196 \pm 171 U / L)$. Não houve alteração nas enzimas ALT e AST. A isoenzima CK-MB e o cTnI alteraram somente em três cães. Os cães infectados naturalmente no semiárido nordestino apresentam características relacionáveis à forma crônica indeterminada, ou seja, cães assintomáticos. A identificação dos cães infectados naturalmente sem características patognomônicas da doença de Chagas ressalta a importância desta enfermidade no processo diagnóstico com as demais que manifestam perfis inespecíficos associados ou não às doenças cardiovasculares.

TERMOS DE INDEXAÇÃO: Cardiopatia, Trypanosoma cruzi, doença de Chagas, doenças de cão, parasitologia.

\section{INTRODUÇÃO}

A doença de Chagas é causada por um protozoário hemoflagelado, Trypanosoma cruzi, e transmitido aos humanos por insetos triatomíneos hematófagos, transplantes de órgãos, transmissão acidental e oral. A infecção através da transfusão de sangue apresenta uma taxa de risco de 12 a $20 \%$ a cada $500 \mathrm{~mL}$ transfundidos. É endêmica em muitas regiões da América Latina, promovendo um risco de $25 \%$ de a população contrair a doença, tendo uma prevalência de 17,4 milhões de casos. (WHO 2002, Carvalho et al. 2003, Gutierrez et al. 2009).

Este parasita tem sido detectado em uma ampla variedade de espécies de animais domésticos e selvagens incluindo cães, gatos, roedores e marsupiais (Brener et al.
2000), sendo o cão considerado o seu principal reservatório doméstico (Montenegro et al. 2002). Cães e gatos são importantes epidemiologicamente no processo de infecção por insetos, no entanto os residentes das casas e os cães são aproximadamente três vezes mais infectados que os gatos (Gurtler et al. 2007).

Em cães e seres humanos, a doença apresenta quatro formas clínicas: aguda, crônica indeterminada, subaguda e crônica determinada; podendo manifestar lesões cardíacas, digestivas e menos comumente a nervosa (Brener et al. 2000, Camacho et al. 2003). No Brasil, relacionando aos dados humanos, a forma indeterminada ou assintomática é mais comum em $50-70 \%$ dos casos, seguido pelas formas cardíacas e digestivas em 10-40\% e 7-11\%, respectivamente (Brener et al. 2000, WHO 2002).

Em relação à tripanossomíase canina, Camacho et al. (2003) citam que já foram descritas manifestações agudas e crônicas dessa enfermidade, sendo a doença aguda facilmente observada em cães jovens, entre cinco dias e seis meses de idade. Os animais afetados desenvolvem infecção generalizada, com lesões extensas, de forma predominante no miocárdio e no sistema nervoso central. Caracteriza-se ainda por anorexia, linfadenopatia generalizada, diarreia, miocardite, podendo ocorrer morte súbita decorrente da arritmia grave.

A doença crônica ocorre oito a 36 meses após a infecção inicial (Lappin 1997) e o paciente pode manter-se assintomático ou com sinais clínicos relacionados aos distúrbios de ritmo cardíaco. Em pacientes humanos, as manifestações clínicas se intensificam, com cardiomegalia leve a moderada, evoluindo para insuficiência cardíaca congestiva acompanhada de aumento cardíaco grave, fenômenos tromboembólicos e arritmias graves nos estágios finais (Rossi \& Mengel 1992).

Segundo Marin-Neto et al. (1999), o uso judicioso de diversos exames laboratoriais como de eletrocardiografia, exame radiográfico torácico e ecocardiografia, visa principalmente indicar a presença de anormalidades anatômicas e funcionais, auxiliando no acompanhamento do grau de acometimento orgânico, da evolução da doença e do possível tratamento.

Desta forma, diante da ausência de dados acerca desta enfermidade em cães da região semiárida do nordeste, objetivou-se com esta pesquisa, evidenciar as alterações clínicas e laboratoriais de cães chagásicos naturalmente infectados, auxiliando assim na caracterização da doença.

\section{MATERIAL E MÉTODOS}

Foram avaliados 10 cães positivos para Trypanosoma cruzi domiciliados nas áreas rurais nos municípios de Patos e Teixeira no estado da Paraíba e Caicó no estado do Rio Grande do Norte. Os cães foram diagnosticados mediante análises sorológicas da RIFI (reagente $\geq 1: 20$ ) e ELISA (diluição 1:160, cut off =0,284); PCR, microscopia direta e hemocultura.

Adicionalmente foram realizados testes sorológicos de imunofluorescência indireta (RIFI - Biomanguinhos) e o enzyme linked immunosorbent assay (ELISA S7) para Leishmania chagasi. Esses exames sorológicos foram empregados como forma de determinar reações cruzadas ou co-infecções. 
Os cães diagnosticados positivos para T. cruzi foram submetidos a exame físico (inspeção de mucosas, linfonodos e auscultação torácica), exames para determinação da frequência cardíaca (FC), utilizando-se eletrocardiógrafo computadorizado (TEB - mod. ECGPC VERSÃO 1.10), calculando-a a partir do intervalo $\mathrm{RR}$, registrado em bat/min; frequência respiratória $(f)$ obtida por meio da contagem dos movimentos costo-abdominais em um minuto (mov/min); temperatura corporal $\left(\mathrm{T}^{\circ} \mathrm{C}\right)$ utilizando um termômetro clínico digital, o qual foi inserido no reto por aproximadamente dez segundos.

Foi realizada avaliação eletrocardiográfica utilizando o ECG, empregado na derivação II, onde foram avaliados a duração e amplitude da onda $\mathrm{P}(\mathrm{ms})$ e $\mathrm{P}(\mathrm{mV})$ respectivamente, a duração do complexo QRS (QRSms), a amplitude da onda R (RmV) e os intervalos entre as ondas P e R (PRms), Q e T (QTms) e entre duas ondas R (RRms).

As pressões arteriais sistólica (PAS), diastólica (PAD) e média (PAM), em mmHg, foram avaliadas empregando-se monitor multiparamétrico (INMAX Color - Instramed Ltda), cujo manguito de pressão foi adaptado na região umeral ou femural.

Para avaliação do tamanho aproximado do coração foi empregado o método do Vertebral Heart Scale System (VHS) (Kealy \& McAllister 2005) mediante as radiografias torácicas, obtidas nas projeções dorso-ventral e laterais direita e esquerda.

Amostras de sangue venoso foram colhidas e acondicionadas em tubos com e sem EDTA, os quais foram encaminhados ao Laboratório de Patologia Clínica do HV/UFCG para realização de hemogramas (eritrograma e leucograma) e a determinação dos parâmetros bioquímicos séricos da atividade enzimática da ureia, creatinina, aspartato aminotransferase (AST), alanino aminotransferase (ALT), proteínas totais (PT), albumina e globulina; creatina quinase (CK), creatina quinase fração MB (CK-MB) e complexo troponina I (cTnI). Com exceção desta, realizada por método de quimioluminescência, as demais foram dosadas utilizando-se o método colorimétrico ${ }^{4}$. As leituras foram conduzidas em analisador semi-automático. As análises dos soros para troponina cardíaca tipo I foram realizadas no Laboratório Maracondi em São Carlos, SP.

Foi realizado exame parasitológico de fezes como forma de controle e avaliação auxiliar de doenças concomitantes no Laboratório de Parasitologia do Hospital Veterinário da UFCG, Campus de Patos.

\section{RESULTADOS E DISCUSSÃO}

Os 10 cães que participaram da avaliação clínico-laboratorial apresentavam idade de 4,2 $\pm 1,4$ anos, machos $(7 / 10)$ e fêmeas $(3 / 10)$, sem raça definida. Ao exame físico (Quadro 1), todos os cães apresentaram-se clinicamente normais (mucosas normocoradas, linfonodos com tamanhos normais e móveis, alertas, apetite normal, escore corporal normal, etc.). Em todos os cães observou-se uma FC normal com ritmo sinusal, com exceção de um cão que apresentou taquicardia sinusal (168 bat/min). Tais achados assemelham-se ao registrado por Guedes et al. (2009), que demonstraram que, diferentemente da fase aguda, todos os cães mostraram normalização dos nódulos linfáticos cervicais, fato também relatado por Andrade \& Andrade (1980) e Klein \& Camacho (1997). Essa ausência de sintomatologia clínica pode ser justificada pelo desconhecimento do

\footnotetext{
${ }^{4}$ Kit Labtest Diagnóstica S.A., Av. Paulo Ferreira da Costa 600, Vista Alegre, Lagoa Santa, MG, Brasil.
}

Quadro 1. Resultados clínicos de cães infectados naturalmente por Trypanosoma cruzi no semiárido nordestino

\begin{tabular}{cccccccccc}
\hline Animais & $\begin{array}{c}\text { Idade } \\
\text { (anos) }\end{array}$ & Sexo & $\begin{array}{c}\text { FC } \\
\text { (bat./min) }\end{array}$ & $\begin{array}{c}\text { FR } \\
\text { (mov./min) }\end{array}$ & $\begin{array}{c}\text { TR } \\
\left({ }^{\circ} \mathrm{C}\right)\end{array}$ & PAS & PAD & PAM & VHS \\
\hline 1 & 5 & $\mathrm{~F}$ & 131 & 40 & 38,7 & 123 & 93 & 102 & 10,8 \\
2 & 4 & $\mathrm{M}$ & 117 & 68 & 39,1 & 158 & 112 & 128 & 10,2 \\
4 & 6 & $\mathrm{M}$ & 102 & 24 & 38,4 & 115 & 79 & 91 & 10,0 \\
5 & 4 & $\mathrm{M}$ & 93 & 32 & 38,5 & 122 & 82 & 97 & 10,0 \\
6 & 3 & $\mathrm{~F}$ & 101 & 24 & 38,2 & 136 & 80 & 104 & 10,0 \\
7 & 2 & $\mathrm{~F}$ & 142 & 32 & 39,0 & 186 & 90 & 119 & 9,0 \\
8 & 2 & $\mathrm{M}$ & 168 & 72 & 39,1 & 145 & 75 & 110 & 10,5 \\
9 & 6 & $\mathrm{M}$ & 87 & 32 & 37,7 & 141 & 95 & 114 & 9,5 \\
10 & 5 & $\mathrm{M}$ & 112 & 24 & 38,2 & 116 & 77 & 91 & 9,0 \\
11 & 5 & $\mathrm{M}$ & 98 & 24 & 39,2 & 140 & 75 & 94 & 10,0 \\
Média & 4,2 & & 115 & 37 & 38,6 & 138 & 86 & 105 & 9,9 \\
\pm SD & 1,5 & & 25 & 18 & 0,5 & 22 & 12 & 13 & 0,6
\end{tabular}

$\overline{\mathrm{FC}}=$ frequência cardíaca, $\mathrm{FR}=$ frequência respiratória, $\mathrm{TR}=$ temperatura retal, PAS = pressão arterial sistólica, PAD = pressão arterial diastólica, PAM = pressão arterial média.

período de infecção natural, virulência dependente do tipo de cepa circulante e pela idade dos animais (Guedes et al. 2007, Souza et al. 2008). A idade média dos cães observada nesta pesquisa correspondia a animais adultos, portanto, torna-se mais difícil que os mesmos desenvolvam a fase aguda da doença à semelhança que ocorre em animais jovens, apresentando, assim, sinais menos evidentes (Camacho et al. 2003). Comparativamente aos humanos, Castro et al. (2001) analisou os pacientes chagásicos crônicos e observou que a maior parte deles permaneceram com a forma clínica inalterada e na forma indeterminada.

As médias dos valores das pressões arteriais (PAS: 138,0 $\pm 22,0 \mathrm{mmHg}$; PAD: 86,0 $\pm 12 \mathrm{mmHg}$; PAM: $105,0 \pm 12,0 \mathrm{mmHg}$ ) apresentaram-se dentro dos padrões de normalidade citados por Camacho et al. (2003). A normalização da pressão arterial em cães chagásicos também foi observada por outros autores (Alves 2003, Souza et al. 2008), podendo se inferir que a idade desses animais pode ter influenciado na manutenção da pressão arterial haja vista que, segundo Bertanha et al. (2008), pacientes humanos chagásicos crônicos com pressão normal apresentavam-se com menos idade que os chagásicos hipertensos. A pressão normal em chagásicos desacelera danos cardiovasculares, reduzindo a progressão da doença para cardiopatias e alterações eletrocardiográficas.

Nenhum dos cães manifestou sinais de congestão (ascite, edema pulmonar, cianose, pulso jugular positivo, efusão pericádica, etc.), os quais são decorrentes de insuficiência cardíaca observada também por Andrade \& Andrade (1980) quando experimentalmente 10 animais evoluíram para a fase crônica indeterminada da infecção.

As projeções realizadas para avaliação radiográfica dos cães não demonstraram nenhuma alteração e isso contrasta com a avaliação eletrocardiográfica, cujos valores sugerem, de acordo com Tilley \& Goodwin (2002), aumento atrial por apresentarem Pms com média $47 \pm 6,5 \mathrm{~ms}$. A elevação na duração da onda $\mathrm{P}$ em cães chagásicos sem aumento na silhueta cardíaca também foi registrado por Souza et al. (2008), contradizendo os achados de Klein \& Camacho (1997) e Pascon et al. (2010).

As alterações eletrocardiográficas (Quadro 2) observadas neste estudo foram discretas e com poucas exceções 
Quadro 2. Resultados eletrocardiográficos de cães infectados naturalmente por Trypanosoma cruzi no semiárido nordestino

\begin{tabular}{ccccccccc}
\hline Animais & PmV & Pms & PR & QRS & RR & RmV & QT & T (<25\%) \\
\hline 1 & 0,17 & 47 & 100 & 30 & 913 & 0,44 & 187 & $<25 \%$ \\
2 & 0,13 & 50 & 120 & 37 & 1020 & 0,79 & 180 & $<25 \%$ \\
4 & 0,12 & 40 & 130 & 37 & 1173 & 1,04 & 210 & $<25 \%$ \\
5 & 0,16 & 47 & 133 & 30 & 1277 & 0,57 & 200 & $<25 \%$ \\
6 & 0,2 & 60 & 123 & 30 & 1183 & 0,54 & 227 & $<25 \%$ \\
7 & 0,12 & 47 & 100 & 33 & 840 & 0,45 & 173 & $<25 \%$ \\
8 & 0,15 & 53 & 123 & 33 & 713 & 1,13 & 180 & $<25 \%$ \\
9 & 0,1 & 43 & 97 & 30 & 1357 & 0,54 & 217 & $<25 \%$ \\
10 & 0,13 & 47 & 110 & 40 & 1063 & 1,14 & 210 & $<25 \%$ \\
11 & 0,22 & 37 & 103 & 30 & 1147 & 0,75 & 197 & $<25 \%$ \\
Média & 0,15 & 47 & 114 & 33 & 1.069 & 1 & 198 & \\
\pm SD & 0,0 & 6,5 & 13,5 & 3,7 & 200,6 & 0,3 & 17,9 &
\end{tabular}

$\mathrm{PmV}=$ amplitude da onda $\mathrm{P}$ em milivolts, $\mathrm{Pms}=$ duração da onda $\mathrm{P}$ em milisegundos, $\mathrm{PR}=$ intervalo entre as ondas $\mathrm{P}$ e $\mathrm{R}$ em milisegundos, $\mathrm{QRS}$ = duração do complexo QRS em segundos, $\mathrm{RR}=$ intervalo entre duas ondas R em milisegundos, $\mathrm{RmV}=$ amplitude da onda R em milivolts, $\mathrm{QT}$ = duração do intervalo QT em milisegundos, $\mathrm{T}=$ amplitude da onda $\mathrm{T}$.

estiveram dentro dos limites da normalidade. Outrossim, estudos experimentais observam que a cronicidade da infecção leva à redução ou desaparecimento de alterações no sistema excito-condutor, principalmente as arritmias (Barr et al. 1992, Souza et al. 2008), fato também observado em humanos (Castro et al. 2001).

Foi observado no cão C8 supradesnivelamento do segmento ST, que variou, nos três momentos registrados, de 0,16 a $0,23 \mathrm{mV}$. Apesar de ter se registrado supradesnivelamento deve-se salientar que este achado não pode ser caracterizado como oriunda de lesões típicas do miocárdio, como hipóxia e isquemia (Tilley \& Goodwin 2002), haja vista que os marcadores empregados para identificar lesões cardíacas, a isoenzima CK-MB e cTnI, mantiveram-se normais.

Em relação às variáveis hematológicas, $60 \%$ e $70 \%$ dos cães apresentaram trombocitopenia e anemia, respectivamente. Os valores médios da hemoglobina e hematócrito estiveram abaixo dos limites de normalidade (Quadro 3). Os animais cães valores médios de trombócitos, eritrócitos, hemoglobina e hematócrito abaixo dos limites considerados normais para a espécie, tendo a maioria apresentado anemia do tipo normocítica normocrômica, como relatado

\section{Quadro 3. Resultados do eritrograma de cães infectados naturalmente por Trypanosoma cruzi no semiárido nordestino}

\begin{tabular}{ccccccc}
\hline Animais & $\begin{array}{c}\text { Eritrócito } \\
(\mathrm{uL})\end{array}$ & $\begin{array}{c}\mathrm{Hb} \\
(\mathrm{g} / \mathrm{dL})\end{array}$ & $\begin{array}{c}\mathrm{Ht} \\
(\%)\end{array}$ & $\begin{array}{c}\mathrm{VGM} \\
(\mathrm{fL})\end{array}$ & $\begin{array}{c}\text { CHCM } \\
(\%)\end{array}$ & $\begin{array}{c}\text { PLAQ } \\
\left(\mathrm{mm}^{3}\right)\end{array}$ \\
\hline 1 & 3.750 .000 & 8,5 & 24 & 64 & 35,41 & 52.000 \\
2 & 4.840 .000 & 10,3 & 33 & 68,7 & 31,2 & 62.400 \\
4 & 4.630 .000 & 11,8 & 31 & 67,3 & 38 & 101.000 \\
5 & 4.750 .000 & 9,1 & 23 & 48,9 & 21,1 & 127.000 \\
6 & 7.100 .000 & 13,9 & 43 & 60,5 & 32,3 & 390.000 \\
7 & 4.800 .000 & 10,1 & 31 & 64,5 & 32,5 & 437.000 \\
8 & 5.800 .000 & 12 & 35 & 60,3 & 34,2 & 186.000 \\
9 & 5.140 .000 & 12 & 37 & 72,5 & 32,4 & 128.000 \\
10 & 5.900 .000 & 15,1 & 50 & 84,7 & 15,1 & 289.000 \\
11 & 3.720 .000 & 10,8 & 33 & 89,1 & 32,7 & 102.000 \\
Média & 5.043 .000 & 11 & 34 & 68 & 30 & 187.440 \\
\pm SD & 1.018 .005 & 2,0 & 8,1 & 12 & 7,0 & 137.261
\end{tabular}

$\mathrm{Hb}=$ hemoglobina, $\mathrm{Ht}=$ hematócrito, $\mathrm{VCM}=$ volume corpuscular médio, $\mathrm{CHCM}=$ concentração de hemoglobina corpuscular média, $\mathrm{PLAQ}=$ plaquetas.

por Rashid et al. (2008). Por outro lado, Klein \& Camacho (1997) verificaram tais achados apenas na fase aguda da doença. A trombocitopenia também foi observada em cães por Lana et al. (1989) e em macacos por Cardoso \& Brener (1980). Esta redução de plaquetas na corrente sanguínea sugere que a causa possa ser decorrente, dentre outras causas, de uma deficiência plaquetária primária com a participação de imuno-complexos ou sistema complemento, do tipo de cepa, bem como da parasitemia por T. cruzi (Cardoso \& Brener 1980). Importante registrar que em dois cães deste estudo (C1 e C4) houve infecção concomitante por Babesia sp. e Anaplasma platys que podem ter agravado o quadro plaquetário.

$\mathrm{Na}$ avaliação do leucograma observaram-se valores médios dentro dos limites de normalidade, com exceção dos eosinófilos, cujas médias registradas estavam elevadas (Quadro 4).

A eosinofilia observada em três cães determinou a elevação da média geral do grupo estudado. Tal elevação dos eosinófilos ocorreu pela presença de parasitas intestinais em dois destes cães com eosinofilia, devido a essas células serem responsáveis pelo mecanismo de defesa contra os estágios larvários de parasitas (Trall et al. 2006). Adicionalmente, a eosinofilia está relacionada às respostas imunes

Quadro 4. Resultados do leucograma realizado de cães infectados naturalmente por Trypanosoma cruzi no semiárido nordestino

\begin{tabular}{|c|c|c|c|c|c|c|c|c|c|c|c|c|}
\hline Animais & LEUC (uL) & NS (\%) & NS (Abs) & NB (\%) & NB (Abs) & EOS (\%) & EOS (Abs) & LINF (\%) & LINF (Abs) & MON (\%) & MON (Abs) & BAS (\%) \\
\hline 1 & 9.000 & 91 & 8190 & 0 & 0 & 0 & 0 & 7 & 630 & 2 & 180 & 0 \\
\hline 2 & 11.200 & 58 & 6.496 & 0 & 0 & 7 & 784 & 29 & 3.248 & 6 & 672 & 0 \\
\hline 4 & 15.200 & 65 & 9.880 & 0 & 0 & 3 & 456 & 23 & 3.496 & 5 & 625 & 0 \\
\hline 5 & 5.900 & 61 & 3.599 & 0 & 0 & 5 & 295 & 32 & 1.888 & 1 & 59 & 0 \\
\hline 6 & 11.800 & 58 & 6.844 & 0 & 0 & 26 & 3.068 & 14 & 1.652 & 2 & 236 & 0 \\
\hline 7 & 29.950 & 58 & 17.371 & 0 & 0 & 30 & 8.985 & 12 & 3.594 & 0 & 0 & 0 \\
\hline 8 & 10.750 & 84 & 9.030 & 2 & 215 & 1 & 108 & 10 & 1.080 & 3 & 323 & 0 \\
\hline 9 & 21.400 & 49 & 10.486 & 1 & 214 & 6 & 1.284 & 42 & 8.988 & 2 & 428 & 0 \\
\hline 10 & 16.400 & 55 & 9.200 & 1 & 164 & 28 & 4.756 & 15 & 2.460 & 1 & 164 & 0 \\
\hline 11 & 17.650 & 51 & 9.270 & 0 & 0 & 5 & 885 & 43 & 7.611 & 1 & 177 & 0 \\
\hline Média & 14.925 & 63 & 9.037 & 0,4 & 59,3 & 11 & 2.062 & 23 & 3.465 & 2 & 286 & 0 \\
\hline$\pm \mathrm{SD}$ & 6952 & 14 & 3557 & 1 & 96 & 12 & 2860 & 13 & 2754 & 2 & 226 & 0 \\
\hline
\end{tabular}

NS = neutrófilo segmentado, NB = bastonetes, EOS = eosinófilos, LINF = linfócitos, MON = monócitos, BAS = bastonetes, Abs = valor absoluto, $\%$ = valor relativo. 
Quadro 5. Resultados das análises bioquímicas de cães infectados naturalmente por Trypanosoma cruzi no semiárido nordestino

\begin{tabular}{|c|c|c|c|c|c|c|c|c|c|c|}
\hline Animais & Ureia $(\mathrm{g} / \mathrm{dL})$ & Creat. (mg/dL) & PT (d/dL) & Alb. (g/dL) & Glob. (g/dL) & $\operatorname{ALT}(\mathrm{U} / \mathrm{mL})$ & AST (U/dL) & $\mathrm{CK}(\mathrm{U} / \mathrm{L})$ & CK-MB (U/L) & cTnI (ng/mL) \\
\hline 1 & 34 & 0,7 & 8,1 & 1,69 & 6,41 & 47 & 26 & 72 & 14,2 & 0,06 \\
\hline 2 & 46 & 0,9 & 8,2 & 1,74 & 6,46 & 26 & 41 & 97 & 24,0 & 0,06 \\
\hline 4 & 36 & 0,8 & 7,1 & 2,34 & 4,76 & 31 & 20 & 72 & 31,2 & 0,06 \\
\hline 5 & 25 & 1,0 & 7,1 & 1,88 & 5,22 & 20 & 36 & 145 & 31,8 & 0,06 \\
\hline 6 & 40 & 1,0 & 6,6 & 3,01 & 3,59 & 52 & 26 & 72 & 13,2 & 0,06 \\
\hline 7 & 24 & 0,9 & 5,5 & 2,07 & 3,43 & 31 & 36 & 315 & 47,4 & 0,06 \\
\hline 8 & 24 & 0,8 & 8,4 & 2,56 & 5,84 & 31 & 115 & 607 & 56,4 & 0,06 \\
\hline 9 & 40 & 1,1 & 7,1 & 2,36 & 4,74 & 26 & 20 & 121 & 14,6 & 0,06 \\
\hline 10 & 32 & 1,0 & 6,8 & 1,88 & 4,92 & 52 & 31 & 145 & 22,0 & 0,06 \\
\hline 11 & 37 & 1,0 & 7,5 & 2,13 & 5,37 & 83 & 31 & 315 & 52,8 & 0,06 \\
\hline Média & 34 & 0,9 & 7,2 & 2,2 & 5,1 & 40 & 38 & 196 & 30,8 & 0,06 \\
\hline$\pm \mathrm{SD}$ & 7,6 & 0,1 & 0,9 & 0,4 & 1,0 & 19 & 28 & 171 & 16,3 & 0,0 \\
\hline
\end{tabular}

Creat. = creatinina, $\mathrm{PT}=$ proteína total, Alb. = albumina, Glob.= globulina, ALT = alanina aminotransferase, $\mathrm{AST}=\mathrm{aspartato}$ aminotransferase, CK $=$ creatinoquinase, $\mathrm{CK}-\mathrm{MB}=$ creatinoquinase isoenzima $\mathrm{MB}, \mathrm{cTnI}=$ complexo troponina $\mathrm{I}$.

dos cães chagásicos crônicos (Lana et al. 1989) aos parasitas de T.cruzi (Guedes et al. 2009).

Três cães apresentaram leucocitose, dois com linfocitose e outro com neutrofilia. Esses achados também foram observados por Lana et al.(1989) e Barr et al. (1991) em cães tanto na fase aguda como crônica e estão relaciondos à inflamação decorrida da infecção por Trypanosoma cruzi.

$\mathrm{Na}$ avaliação bioquímica (Quadro 5), a ureia e a creatinina apresentaram-se com valores individuais e médios nos padrões normais, no entanto, os valores médios demonstraram uma hiperproteinemia, hipoalbuminemia e hiperglobulinemia. Essa hiperproteinemia também registrada em tamarins leão dourado (Monteiro et al. 2006), sugere um provável reflexo da resposta humoral causada pela infecção por T. cruzi, confirmado pela hiperglobulinemia. Assim como nos humanos, nos cães após a fase aguda decrescem também os níveis de imunoglobulinas da classe M e sobem os níveis de IgG, que com a cronicidade da infecção inicia o decréscimo e tendem a estabilizar, auxiliando na identificação da transição para a fase crônica (Lana et al. 1992, Brener et al. 2000, Guedes et al. 2002).

A atividade sérica da enzima $\mathrm{CK}$ alcançou valores médios e individuais acima dos valores de referência $(196 \pm 171 \mathrm{U} / \mathrm{L})$ citados por Lopes et al. (2005). 0 mesmo não ocorreu com as enzimas ALT, AST e os marcadores cardíacos. No entanto, individualmente, valores de CK-MB dos cães C7, C8 e C11 alcançaram uma dosagem acima da referência. As médias de cTnI também foi registrada nos limites de normalidade, segundo Sleeper et al. (2001) e Santos (2005). O valor médio da atividade enzimática da $\mathrm{CK}$ apresentou-se aumentado, mas não foi observado nenhum comprometimento muscular, ósseo ou hepático nos cães deste estudo que justificassem tal elevação, visto que as médias de ALT e AST encontravam-se normais. Estes achados diferem daqueles obtidos por Barr et al. (1991), que registraram essas enzimas em concentrações altas. Em relação aos resultados obtidos para CK-MB neste estudo, os mesmos estão de acordo com os obtidos por Monteiro et al. (2006), mas divergem daqueles registrados por Souza et al. (2008) em cães naturalmente infectados.

Apesar de apenas a enzima CK ter apresentado médias acima dos limites da normalidade e somente três cães individualmente alcançarem atividade enzimática de CK-MB acima do normal, pode-se inferir que o parasitismo tecidu- al do T. cruzi no tecido miocárdico não seja a principal causa de miocardite. Ela também pode ser decorrente de outros mecanismos, como a resposta imunológica, incluindo a celular e humoral (Pascon et al. 2010), com consequentes lesões miocárdicas tanto na fase aguda como crônica (Rossi \& Mengel 1992). Nos cães que apresentaram valores de CK e CK-MB acima dos níveis normais pode sugerir lesão cardíaca (Souza et al. 2008) e, apesar, da dosagem por quimioluminescência de cTnI, ser considerada mais sensível e específica que a CK-MB, esta apresentou valores normais devido sua maior presteza em diagnóstico de lesões agudas que nas crônicas (Tarducci et al. 2004).

\section{CONCLUSÃO}

Os cães infectados naturalmente no semiárido nordestino apresentam características relacionáveis à forma crônica indeterminada, ou seja, animais assintomáticos. A ausência de manifestações clínicas evidentes da doença de Chagas ressalta a importância desta enfermidade no processo de diagnóstico diferencial com as demais que manifestam perfis inespecíficos, associados ou não a doenças cardiovasculares.

Agradecimentos.- À Secretaria de Saúde do Estado da Paraíba, à Prof ${ }^{\mathrm{a}}$ Lúcia Maria C. Galvão da Faculdade de Farmácia da Universidade Federal do Rio Grande do Norte e à Coordenação de Aperfeiçoamento de Pessoal de Nível Superior (CAPES) pela concessão da bolsa de estudos.

\section{REFERÊNCIAS}

Alves R.O. 2003. Avaliações ecodopplercardiográfica, eletrocardiográfica computadorizada e dinâmica (Sistema Holter) e clinico-patológica em cães com cardiopatia chagásica experimental. Tese de Doutorado em Medicina Veterinária, Faculdade de Ciências Agrárias e Veterinária, Universidade Estadual Paulista, Jaboticabal, SP. 89p.

Andrade Z.A. \& Andrade S.G. 1980. A patologia da doença de chagas experimental no cão. Mem. Inst. Oswaldo Cruz 75(3/4):77-95.

Barr S.C., Gosset K.A. \& Klei T.R. 1991. Clinical, clinicopathologic, and parasitologic observation of trypanosomiasis in dog infected with North American Trypanosoma cruzi isolates. Am. J. Vet. Res. 52(6):954-960.

Barr S.C., Holmes R.A. \& Klei T.R. 1992. Electrocardiographic and echocardiographic features of trypanosomiasis in dog inoculated with North American Trypanosoma cruzi isolates. Am. J. Vet. Res. 53(4):521-527.

Bertanha L., Guariento M.E., Magna L.A. \& Almeida E.A. 2008. Caracterização clínico-laboratorial de chagásicos hipertensos sem insuficiência cardíaca manifesta. Revta Soc. Bras. Med. Trop. 41(2):163-168. 
Brener Z., Andrade Z.A. \& Barral-Neto M. 2000. Trypnosoma cruzi e doença de Chagas. $2^{\underline{a}}$ ed. Guanabara Koogan, Rio de Janeiro. 430p.

Camacho A.A., Mucha C.J. \& Belerenian G.C. 2003. Afecções Cardiovasculares em Pequenos Animais. Interbook, São Paulo. 328p.

Cardoso J.E. \& Brener Z. 1980. Hematological changes in mice experimentally infected with Trypanosoma cruzi. Mem. Inst. Oswaldo Cruz 75(3/4):97-104.

Carvalho C.M.E., Andrade M.C.R., Xavier S.S., Mangia R.H.R., Britto C.C., Jansen A.M., Fernandes O., Lannes-Vieira J. \& Bonecini-Almeida M.G. 2003. Chronic Chagas' disease in Rhesus monkeys (Macaca mulatta): Evalution of parasitemia, serology, eletrocardiography, echocardiography, and radiology. Am. J. Trop. Med. Hyg. 68(6):683-691.

Castro C., Prata A. \& Macêdo V. 2001. Estudo clínico durante 13 anos de 190 chagásicos crônicos de Mambaí, Goiás, Brasil. Revta Soc. Bras. Med. Trop. 34(4):309-318.

Guedes P.M.M., Veloso V.M., Tafuri W.L., Galvão L.M.C., Carneiro C.M., Lana M., Chiari E., Soares K.A. \& Bahia M.T. 2002. The dog as model for chemotherapy of the Chagas' disease. Acta Tropica 84:9-17.

Guedes P.M.M., Veloso V.M., Caliari M.V., Carneiro C.M., Souza S.M., Lana M., Chiari E., Bahia M.T. \& Galvão L.M.C. 2007. Trypanosoma cruzi high infectivity in vitro is related to cardiac lesions during long-term infection in Beagle dogs. Mem. Inst. Oswaldo Cruz 102(2):141-147.

Guedes P.M.M., Veloso V.M., Afonso L.C.C., Caliari M.V., Carneiro C.M., Diniz L.F., Marques-da-Silva E.A., Caldas I.S., Mata M.A.V., Souza S.M., Lana M., Chiari E., Galvão L.M.C. \& Bahia M.T. 2009. Development of chronic cardiomyopathy in canine Chagas disease correlates with high IFN- $\gamma$, $\mathrm{TNF}-\alpha$, and low IL-10 production during the acute infection phase. Vet. Immunol. Immunopathol. 130:43-52.

Gurtler R.E., Cecere M.C., Lauricella M.A., Cardinal M.V., Kitron U. \& Cohen J.E. 2007. Domestic dogs and cats as sources of Trypanosoma cruzi infection in rural northwestern Argentina. Parasitology 134(1):69-82.

Gutierrez F.R.S., Guedes P.M.M., Gazzinelli R.T. \& Silva J.S. 2009. The role of parasite persistence in pathogenesis of Chagas heart disease. Parasite Immunol. 31:673-685.

Kealy J.K. \& McAllister H. 2005. Radiologia e ultra-sonografia do cão e do gato. 3aㅡ ed. Manole, São Paulo, p.149-226.

Klein R.P. \& Camacho A.A. 1997. Eletrocardiographic evaluation of dogs experimentally infected with Trypanosoma cruzi during the acute and indeterminate chronic phases of infection. Braz. J. Vet. Res. Anim. Sci. 34(6):337-344.

Lana M., Tafuri W.L., Toledo M.J.O. \& Veloso V.M. 1989. Alterações hematológicas em cães infectados com as cepas Be-62 e Be-78 de Trypanosoma cruzi. Revta Soc. Bras. Med. Trop. 22(Supl.):168.

Lana M., Chiari E. \& Tafuri W.L. 1992. Doença de Chagas experimental em cães. Mem. Inst. Oswaldo Cruz 87(1):59-71.
Lopes S.T.A., Franciscato C., Teixeira L.V., Oliveira T.G.M., Garmatz B.C., Veiga A.P.M. \& Mazzanti A. 2005. Determinação da creatina quinase em cães. Revta Fac. Zootec. Vet. Agron., Uruguaiana, 12(1):31-37.

Lappin M.R. 1997. Infecções protozoárias e mistas, p.431-440. In: Ettinger S.J. \& Feldman E.C. (Eds), Tratado de Medicina Veterinária Interna: doenças do cão e do gato. Vol.1. 4⿳亠丷厂 ed. Manole, São Paulo. 1039p.

Marin-Neto J.A., Simões M.V. \& Sarabanda A.V.L. 1999. Cardiopatia chagásica. Arq. Bras. Cardiol. 72(3):247-263.

Monteiro R.V., Baldez J., Dietz J., Baker A., Lisboa C.V. \& Jansen A.M. 2006. Clinical, biochemical, and electrocardiographic aspects of Trypanosoma cruzi infection in free-ranging golden lion tamarins (Leontopithecus rosalia). J. Med. Primatol. 35:48-55.

Montenegro V.M., Jiménez M., Dias J.C.P. \& Zeledón R. 2002. Chagas disease in dogs from endemic áreas of Costa Rica. Mem. Inst. Oswaldo Cruz 97(4):491-494.

Pascon J.P.E., Neto G.B.P., Sousa M.G., Júnior D.P. \& Camacho A.A. 2010. Clinical characterization of chronic chagasic cadiomyopathy in dogs. Pesq. Vet. Bras. 30(2):115-120.

Rashid A., Rasheed K. \& Hussain A. 2008. Trypanosomiasis in dog; a case report. Iranian J. Arthopod-borne Disease 2(2):48-51.

Rossi M.A. \& Mengel J.O. 1992. Patogênese da miocardite chagásica crônica: o papel de fatores autoimunes e microvasculares. Revta Inst. Med. Trop. 34(6):593-599.

Santos A.L.F. 2005. Dosagem sérica da enzima creatinofosfoquinase-isoenzima MB (CK-MB) e de troponina I (cTnI) de cães eletrocardiograficamente normais e naqueles com desníveis (infra e supra) do segmento ST, utilizando ensaio imunométrico por quimioluminescência. Dissertação de Mestrado em Clínica Veterinária, Faculdade de Medicina Veterinária e Zootecnia, USP, São Paulo, SP. 61p.

Sleeper M.M., Clifford C.A. \& Laster L.L. 2001. Cardiac troponin I in the normal dog and cat. J. Vet. Intern. Med. 15:501-503.

Souza A.I., Paulino-Junior D., Sousa M.G. \& Camacho A.A. 2008. Aspectos clínico-laboratoriais da infecção natural por Trypanosoma cruzi em cães de Mato Grasso do Sul. Ciência Rural 38(5):1351-1356.

Tarducci A., Abate O., Borgarelli M., Borrelli A., Zanatta R. \& Cagnasso A. 2004. Serum values of cardiac troponin-T in normal and cardiomyophatic dogs. Vet. Res. Commun. 28:385-388.

Tilley L.P. \& Goodwin J.K. 2002. Manual de Cardiologia para Cães e Gatos. 3a ed. Roca, São Paulo. 489p.

Trall M.A., Baker D.C., Campbell T.W., DeNicola D., Fettman M.J., Lassen E.D., Rebar A. \& Weiser G. 2006. Hematologia e Bioquímica Clínica Veterinária. Roca, São Paulo. 582p.

WHO 2002. Control of Chagas disease. World Health Organization Technical Report Series 905: i-vi, 1-109. 